\title{
miR-221 inhibits autophagy and targets TP53INP1 in colorectal cancer cells
}

\author{
DAN LIAO ${ }^{1,2^{*}}$, TONG LI $^{*}$, CAIGUO YE ${ }^{1 *}$, LIUYAN ZENG $^{3}$, HUAHUI LI $^{1}$, XINGXIANG PU ${ }^{4}$, \\ CONGCONG DING $^{1}$, ZHIWEI HE $^{1}$ and GUO-LIANG HUANG ${ }^{1}$
}

${ }^{1}$ China-American Cancer Research Institute, Dongguan Scientific Research Center,

Key Laboratory for Epigenetics of Dongguan City, Guangdong Provincial Key Laboratory of Medical Molecular Diagnostics, Guangdong Medical University, Dongguan, Guangdong 523808; ${ }^{2}$ Department of Gynaecology and Obstetrics,

Dongguan Third People's Hospital, Affiliated Dongguan Shilong People's Hospital of Southern Medical University, Dongguan, Guangdong 523326; ${ }^{3}$ Department of Health Management Center, The Affiliated Hospital of Guangdong Medical University, Zhanjiang, Guangdong 524001; ${ }^{4}$ Department II of Chest Internal Medicine, Hunan Cancer Hospital The Affiliated Cancer Hospital of Xiangya School of Medicine, Central South University, Changsha, Hunan 410013, P.R. China

Received June 29, 2017; Accepted November 15, 2017

DOI: 10.3892/etm.2017.5522

\begin{abstract}
Colorectal cancer (CRC) is the third most common cancer and the fourth leading cause of cancer-associated mortalities worldwide. MicroRNAs (miRNAs/miRs) serve important roles in tumor development, progression and metastasis. miR-221 has been reported to modulate proliferation, apoptosis, cell cycle distribution and cell migration in a variety of cancers. However, the function of miR-221 in the autophagy of cancer is unclear. In the present study, the role of miR-221 in the autophagy of CRC cells was investigated and its associated target was identified. Survival analysis using The Cancer Genome Atlas data suggested that a higher expression of miR-221 was associated with poor survival in patients with CRC. A Cell Counting kit-8 assay revealed that miR-221 promoted CRC cell proliferation. Autophagy flux analyzed by microtubule-associated protein 1 light chain 3 (LC3) turnover indicated that miR-221 reduced autophagy
\end{abstract}

Correspondence to: Dr Guo-Liang Huang or Professor Zhiwei He, China-American Cancer Research Institute, Dongguan Scientific Research Center, Guangdong Medical University, 1 Xincheng Road, Dongguan, Guangdong 523808, P.R. China

E-mail: huangguoliang@gdmu.edu.cn; guolianghuang@foxmail.com E-mail: zhiweihe688@yahoo.com

*Contributed equally

Abbreviations: CRC, colorectal cancer; miRNAs, microRNAs; TP53INP1, tumor protein 53-induced nuclear protein 1; TCGA, The Cancer Genome Atlas; LC3, microtubule-associated protein 1 light chain 3

Key words: autophagy, colorectal cancer, miR-221, tumor protein 53 -induced nuclear protein 1 in CRC cells using different protease inhibitors (E64d and pepstatin A; Bafilomycin A1) in nutrient-rich medium or under starvation conditions. Tumor protein 53-induced nuclear protein 1 (TP53INP1) was identified as a potential novel target of miR-221 by bioinformative prediction. The protein expression of TP53INP1 was inversely regulated by miR-221 in CRC cells. Furthermore, luciferase activity assays were performed and indicated that miR-221 may regulate the luciferase activity of wild-type TP53INP1 without interfering with the activity of mutant TP53INP1. These data suggested that miR-221 may promote the cell proliferation of CRC via the inhibition of autophagy and targeted TP53INP1.

\section{Introduction}

Colorectal cancer (CRC) is the third most common cancer and the fourth leading cause of cancer-associated death worldwide (1). The morbidity rates of CRC are increasing substantially in a number of countries within Eastern Asia and Eastern Europe which were previously at low risk (2). The multifactorial etiology of CRC involves lifestyle and dietary factors, such as smoking, red and processed meat consumption, and excessive alcohol consumption (3). Autophagy is a vital transformational switch among mechanisms that are involved in the pathogenesis of CRC (4). Autophagy may act as a suppressor during early stages and as a promoter during advanced stages of CRC $(4,5)$. It is important to determine the regulative mechanisms of autophagy in CRC.

Recent studies suggests that the post-transcription and translation regulation mediated by microRNAs (miRNAs/miRs) contribute significantly to autophagy in cancer (6). It is found that miR-23b-3p inhibits autophagy in gastric cancer cells (7) and miR-26 suppresses autophagy in hepatocellular carcinoma cells (8). Whereas miR-193b is suggested to induce autophagy in oesophageal cancer cells (9). It is interesting that different miRNAs play diverse roles in the regulation of autophagy through various targets. 
Recently, miR-221 is indicated to inhibit autophagy activity by modulating the p27/CDK2/mTOR axis in heart failure (10). Upregulation of miR-221 is also found to inhibit hypoxia/re-oxygenation induced autophagy (11). Inhibition of autophagy by miR-221 in human umbilical vein endothelial cells is further reported (12). Although miR-221 is found to regulate autophagic gene in cancer (13), no direct evidence suggests a role of miR-221 in cancer related autophagy (14). In this study, we demonstrated that miR-221 inhibited autophagy activity in CRC cells and targeted tumor protein 53 -induced nuclear protein 1 (TP53INP1), a regulator of autophagy (15).

\section{Materials and methods}

Expression of miR-221 in CRC using The Cancer Genome Atlas (TCGA) data. TCGA, launched by the National Institute of Health (NIH), is a public funded project that create a comprehensive 'atlas' of cancer genomic profiles (16). miRNA data were extracted from TCGA (http://tcga-data.nci.nih.gov/) for CRC tumors (accessed May, 2015). Level 3 Illumina miRNASeq patients with complete follow-up information were used for miRNA expression analysis. The reads per million miRNAs mapped data unit was evaluated for miR-221. The expression of miR-221 was divided into two groups according to the mean expression level.

Cell culture. Human colon cancer cell line CACO2, HT29, HCT116 and SW620 were purchased from the Cell Bank of Type Culture Collection of Chinese Academy of Sciences (Shanghai, China). Cells were cultured in DMEM medium supplemented with $10 \%$ fetal bovine serum (both from Gibco; Thermo Fisher Scientific, Inc., Waltham, MA, USA) and maintained at $37^{\circ} \mathrm{C}$ in a humidified cell incubator of $5 \%$ $\mathrm{CO}_{2}$.

RNA extraction and quantitative reverse transcription polymerase chain reactions. Total RNA was isolated from cultured cells using TRIzol Reagent (Invitrogen; Thermo Fisher Scientific, Inc.). First-strand cDNA were synthesized from $1 \mu \mathrm{g}$ of total RNA using MMLV reverse transcription kit according to the manufacturer's protocol (Promega Corporation, Madison, WI, USA) with specific reverse transcription primer. Real-time quantitative PCR analysis was performed using FS Universal SYBR-Green Master reagents (Roche Diagnostics, Indianapolis, IN, USA) in an Applied Biosystems PRISM 7500 instruments (Applied Biosystems; Thermo Fisher Scientific, Inc.). The primers for miR-221 were as follows: Reverse transcription, 5'-GTCGTATCC AGTGCAGGGTCCGAGGTATTCGCACTGGATACGACG AAACC-3'; forward, 5'-CGAGCTACATTGTCTGCTGGG T-3' and reverse, 5'-GTGCAGGGTCCGAGGT-3'. U6 RNA was used as an internal control for miRNA normalization. Primer set for U6 was purchased from RiboBio Co., Ltd. All samples were run in triplicate and the $2^{-\Delta \Delta C T}$ method was applied to quantify the relative expression of miR-221.

Plasmids, cell transfection and Cell Counting kit-8 (CCK-8) assay. Overexpression and down-expression plasmids of
miR-221 plus negative control plasmids were purchased from GenePharma Co., Ltd. (Shanghai, China) Transfection of cell lines was carried out using jetPEI (Polyplus-transfection), according to the manufacturer's instructions. Cells with specific transfection were seeded in 96-well plates. CCK-8 solution was added at particular periods of time and incubated for $1 \mathrm{~h}$ at $37^{\circ} \mathrm{C}$. Absorbance was detected at $450 \mathrm{~nm}$ using Synergy ${ }^{2}$ Multi-Mode Microplate Reader (BioTek, Winooski, VT, USA).

Western blot and autophagy flux analysis. Cells were lysed with RIPA buffer (Beyotime Institute of Biotechnology, Haimen, China) and protein concentration was assessed by the BCA assay (Pierce; Thermo Fisher Scientific, Inc.). Equal amount of proteins were separated on SDS-PAGE and transferred to a polyvinylidene difluoride (PVDF) membrane (EMD Millipore, Billerica, MA, USA). The membrane was incubated with the rabbit anti-LC3 II monoclonal antibody (1:1000, \#2775; Cell Signaling Technology, Inc., Danvers, MA, USA) or the rabbit anti-TP53INP1 polyclonal antibody (1:500, sc-68919; Santa Cruz Biotechnology, Inc., Dallas, TX, USA) overnight at $4^{\circ} \mathrm{C}$ and then with goat anti-rabbit IgG infrared-dye-conjugated secondary antibodies (1:10,000, 142801A; Abbkine Scientific Co., Ltd., Wuhan, China) for $1 \mathrm{~h}$ at room temperature. $\beta$-actin $(1: 1,000$, sc-376421; Santa Cruz Biotechnology, Inc.) was used as a loading control. Protein bands were detected by Odyssey Infrared Imaging system (LI-COR Biosciences, Lincoln, NE, USA).

Autophagy flux was measured with LC3II turnover using protease inhibitors, cells were cultured in nutrient-rich medium (DMEM containing 10\% FBS) or starvation conditions (DMEM without FBS) in the absence or presence of E64d (E8640, $10 \mu \mathrm{g} / \mathrm{ml}, 4$ h; Sigma-Aldrich; Merck KGaA, Darmstadt, Germany) and pepstatin A (P5318, $10 \mu \mathrm{g} / \mathrm{ml}, 4 \mathrm{~h}$; Sigma-Aldrich; Merck KGaA), or Bafilomycin A1 (ab120497, $0.1 \mu \mathrm{M}, 2 \mathrm{~h}$; Abcam, Cambridge, UK). Expression of LC3II was quantified and the ratio of LC3II between cells treated without/with protease inhibitors was applied to represent autophagy flux (17).

miR-221 target gene prediction and luciferase reporter assay. TargetScan 6.2 (18), miRanda-rel 2010 (19), and RNA22 v2.0 (20) were applied for bioinformatic analysis. The binding sites were further predicted. The human 3'UTR of TP53INP1 and a mutant of the putative binding site to the seed sequence of miR-221 were cloned into the pGL3-control vector (Promega Corporation) downstream of the firefly luciferase gene to generate the vector pGL3-TP53INP1-WT and pGL3-TP53INP1-MUT by GenePharma Co., Ltd. For the luciferase reporter assay, cells were co-transfected with pGL3-TP53INP1-WT or pGL3-TP53INP1-MUT, and the pRL-TK vector (as calibration) using jetPEI (Polyplus-transfection). Luciferase activity was determined with the Dual Luciferase Reproter Assay system (Promega Corporation).

Statistical analysis. Quantitative data was presented as mean \pm standard deviation (SD). Student's t-test was applied to compare the mean value of each group. Overall survival was estimated with Kaplan-Meier method. Results were 

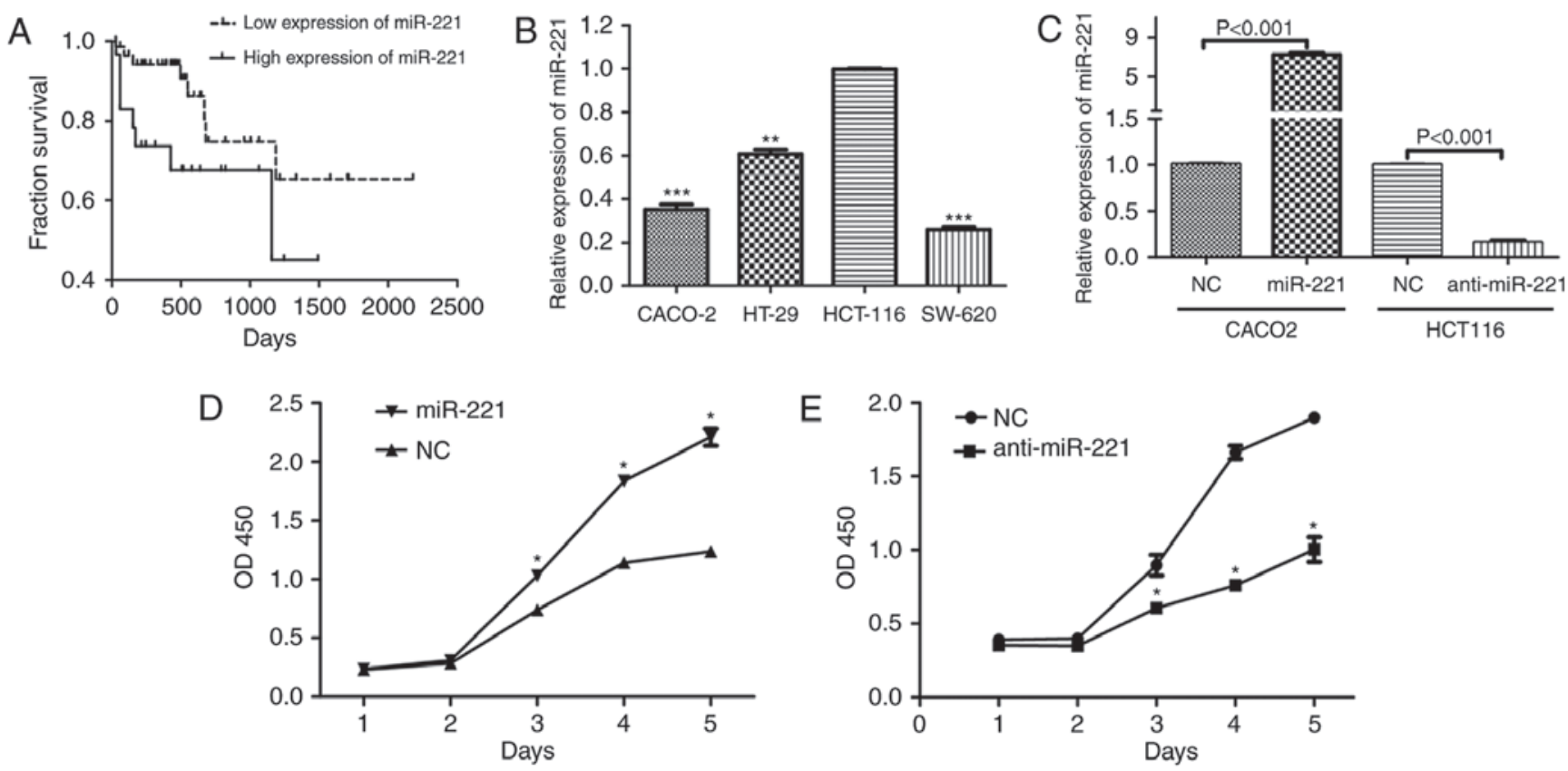

Figure 1. Expression of miR-221 and CRC survival. (A) Association between miR-221 expression and CRC survival was estimated by performing Kaplan-Meier curve analysis using The Cancer Genome Atlas data. Log-rank test, $\mathrm{P}=0.059$; Breslow test, $\mathrm{P}=0.027$; Tarone Ware test, $\mathrm{P}=0.031$. (B) Relative mRNA expression of miR-221 in CRC cells. ${ }^{* *} \mathrm{P}<0.01$ and ${ }^{* * *} \mathrm{P}<0.001$ vs. HCT116 cells. (C) The mRNA expression of miR-221 in transfected CRC cells. $\mathrm{P}<0.001$ as indicated. (D) Overexpression of miR-221 promoted cell growth in CACO2 cells. ${ }^{*} \mathrm{P}<0.05$ vs. NC. (E) Downregulation of miR-221 suppressed cell growth in HCT116 cells. ${ }^{*} \mathrm{P}<0.05$ vs. NC. miR, microRNA; CRC, colorectal cancer; NC, negative control.

considered statistically significant at $\mathrm{P}<0.05$. All analyses were performed using GraphPad Prism (GraphPad Software, Inc., La Jolla, CA, USA).

\section{Results}

miR-221 regulates survival of CRC. miRNA data for CRC patients from TCGA were used to analyze miR-221 affecting CRC patient survival. The result of Kaplan-Meier method indicated that higher expression of miR-221 was associated with worse patient survival (log-rank test, $\mathrm{P}=0.059$; Breslow test, $\mathrm{P}=0.027$; Tarone Ware test, $\mathrm{P}=0.031$; Fig. $1 \mathrm{~A})$. The relative expression of miR-221 was detected in CRC cells. The data showed that the expression of miR-221 was high in HCT116 cells and low in CACO2 and SW620 cells (Fig. 1B). Based on the expression level of miR-221, overexpression of miR-221 in CACO2 and anti-expression in HCT116 cells was implemented respectively in this study. Transfection with miR-221 signifcantly increased the expression of miR-221 in CACO2 cells, while transfection with anti-miR-221 reduced miR-221 expression in HCT116 cells (Fig. 1C). CCK-8 assay was applied to assess miR-221 affecting CRC cells growth. The data suggested that overexpression of miR-221 promoted cell growth in CACO2 cells whereas downregulation of miR-221 inhibited cell growth in HCT116 cells (Fig. 1D and E).

miR-221 impairs autophagy in CRC cells. Autophagy flux analyzed by LC3 turnover was performed to determine autophagy of CRC cells modulated by miR-221. The ratio of LC3II quantified by western blot analysis between cells treated without/with protease inhibitors was used to reflect autophagy flux. The results suggested that LC3II ratio was inversely regulated by miR-221 in both nutrient-rich medium and starvation condition using different protease inhibitors. LC3II ratio was decreased in CACO2 cells with overexpression of miR-221 whereas LC3II ratio was increased in HCT116 cells with downregulation of miR-221 in E64d and pepstatin A (Fig. 2A and B). Similar result was obtained in Bafilomycin A1 treatment, which suggested that LC3II ratio was signifcantly downregulated in $\mathrm{CACO} 2$ cells with overexpression of miR-221 whereas LC3II ratio was increased in HCT116 cells with down-expression of miR-221 (Fig. 2C and D). These data indicated that miR-221 reduced autophagy in CRC cells (Fig. 2).

TP53INP1 is a target of $m i R-221$. Bioinformatic analysis identified TP53INP1 as a potential target of miR-221. Among the predicted targets of hsa-miR-221 by the three programs, 446 miRNA-regulated target genes were identified by Targetscan, 5,670 transcripts were identified by miRanda, and 10,184 transcripts by RNA 22 . We focused our attention on TP53INP1, which is a regulator of autophagy (15). Western blot analysis showed that the protein expression of TP53INP1 was low in HCT116 cells and high in CACO2 and SW620 cells (Fig. 3A). The expression pattern of TP53INP1 protein was inversely correlated with the expression of miR-221. Overexpression of miR-221 in CACO2 cells repressed TP53INP1 expression whereas downregulation of miR-221 in HCT116 cells upregulated TP53INP1 expression (Fig. 3B and C).

The binding sites of TP53INP1 and miR-221 were predicted and luciferase plasmids were constructed and designated as pGL3-TP53INP1-WT and pGL3-TP53INP1-MUT (Fig. 3D). The luciferase activity assay indicated that overexpression of miR-221 suppressed luciferase activity of wild-type TP53INP1 in CACO2 cells whereas downregulation of 
A
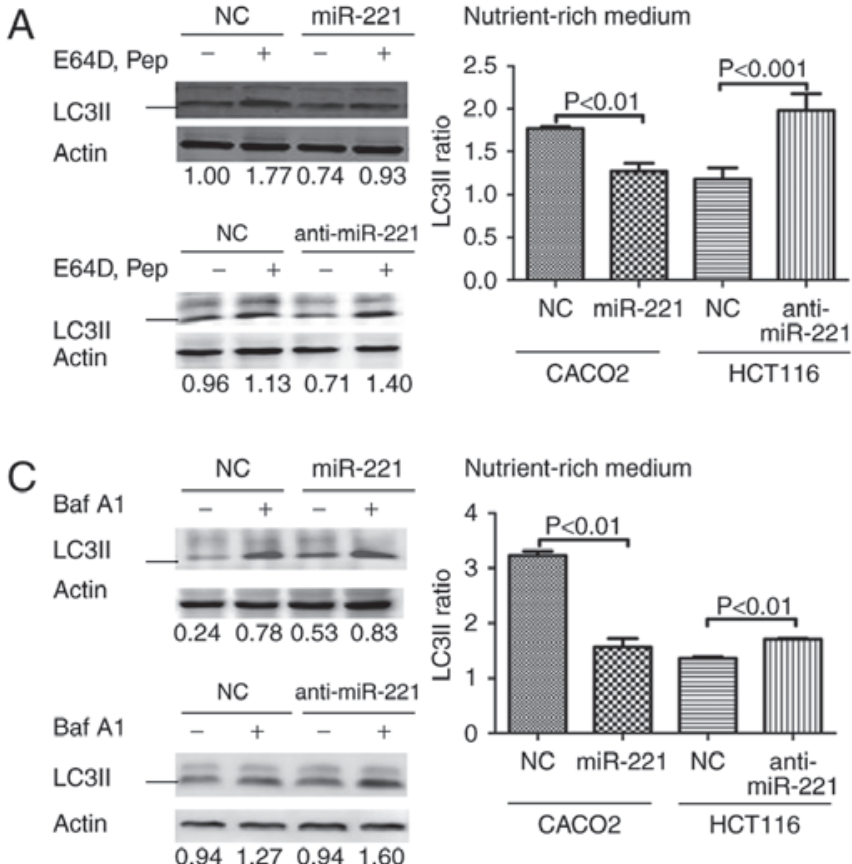

B
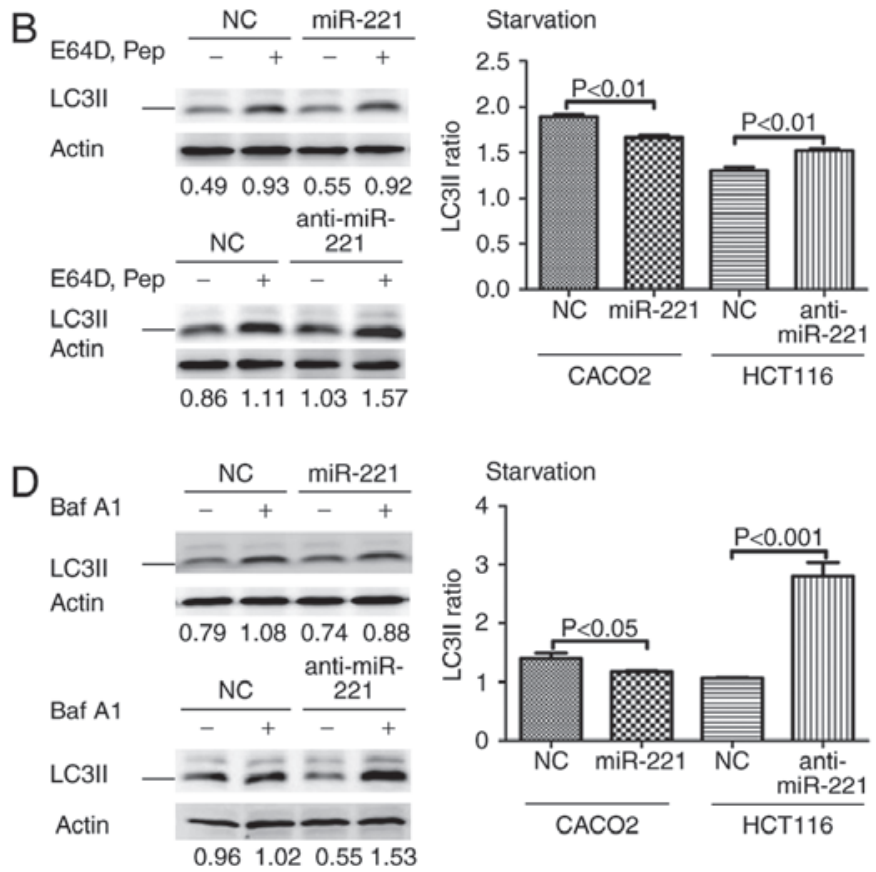

Figure 2. miR-221 inhibits autophagy in CRC cells. Autophagy flux indicated by LC3II turnover was modulated by miR-221 overexpression in CACO2 cells and downexpression in HCT116 cells in different cultivated conditions and drug treatments. (A) Cells were incubated in nutrient-rich medium in the absence (-) or presence (+) of E64d and pepstatin A. (B) Cells were incubated under starvation conditions in the absence (-) or presence (+) of E64d and pepstatin A (C) Cells were incubated in nutrient-rich medium in the absence (-) or presence (+) of Bafilomycin A1. (D) Cells were incubated under starvation conditions in the absence (-) or presence (+) of Bafilomycin A1. $\mathrm{P}<0.05, \mathrm{P}<0.01$ and $\mathrm{P}<0.001$, as indicated. miR, microRNA; CRC, colorectal cancer; $\mathrm{NC}$, negative control; Baf A1, Bafilomycin A1; Pep, pepstatin A.

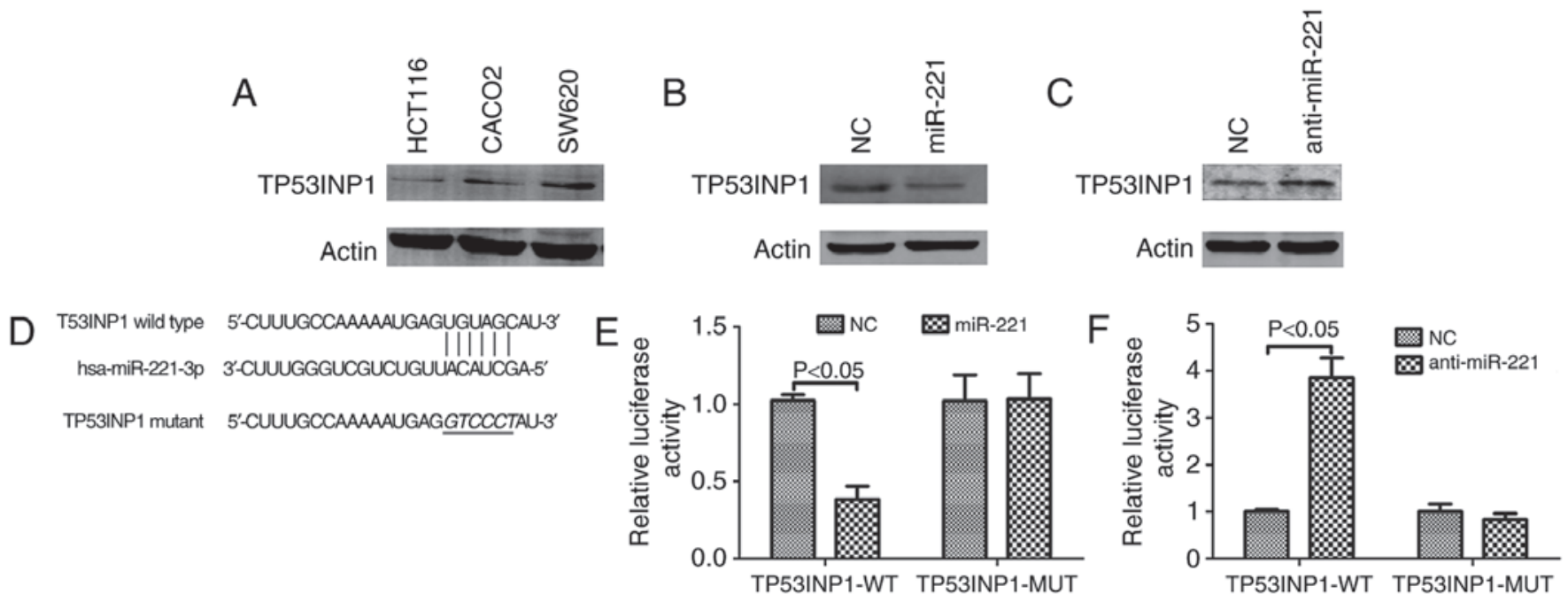

Figure 3. TP53INP1 is a target of miR-221. (A) TP53INP1 protein expression in CRC cells. (B) Overexpression of miR-221 downregulated TP53INP1 protein expression in CACO2 cells. (C) Downregulation of miR-221 upregulated TP53INP1 protein expression in HCT116 cells. (D) WT and MUT of putative miR-221 targeting sequences in TP53INP1 mRNA. (E) Overexpression of miR-221 suppressed luciferase activity of WT TP53INP1 in CACO2 cells. (F) Downregulation of miR-221 increased luciferase activity of WT TP53INP1 in HCT116 cells. P<0.05, as indicated. TP53INP1, tumor protein 53-induced nuclear protein 1; miR, microRNA; CRC, colorectal cancer; NC, negative control; WT, wild-type; MUT, mutant.

miR-221 increased luciferase activity of wild-type TP53INP1 in HCT116 cells without interfering the activity of mutant TP53INP1 (Fig. 3E and F).

\section{Discussion}

The role of miR-221 is considered to act as an oncogene or a tumor suppressor in tumor progression depending on tumor background (21) Overexpression of miR-221 is found in the majority of epithelial tumors (22). miR-221 is a potential biomarker for cancer diagnosis and prognosis (23-25). miR-221 modulates the proliferation, apoptosis, cell cycle distribution and cell migration in a variety of cancers (21). However, the function of miR-221 in autophagy of cancer is not clarified. In this study, we demonstrated that miR-221 inhibited autophagy activity and thereby promoted cell survival in CRC. 
Autophagy is a cellular degradation process by eliminating damaged or superfluous proteins, and unnecessary or dysfunctional cellular components $(26,27)$. Autophagy is a dual function player in cancer progression. On one hand autophagy plays oncosuppressive functions by the clearance of potentially harmful components; on the other hand it facilitates to overcome cellular stress during cancer progression (26). The core pathway of autophagy involves at least five molecular components regulated by a panel of proteins such as Atg1, Atg8, Atg9, Atg12, Beclin 1 and LC3 (28).

Recently, TP53INP1 is found to be a regulator of autophagy (15). TP53INP1 is reported to interact with ATG8-family proteins and LC3 to induce autophagy-dependent cell death (29). Two miRNAs, miR-30a and miR-205, are suggested to suppress autophagy by targeting TP53INP1 $(30,31)$. Our data with bioinformative prediction, expression analysis and luciferase assay indicated that TP53INP1 was a target of miR-221 in CRC cells, which might elucidate the inhibitory effect of autophagy by miR-221. In cardiac remodeling, the inactivation of mTOR abolishes the inhibitory effect of miR-221 on autophagy (10). mTOR as a miR-221 target is also reported in hepatocellular carcinoma cells (32). Overexpression of p27 is found to rescue the impairment of miR-221 on autophagic flux in cardiac remodeling (10). In human umbilical vein endothelial cells, miR-221 is found to inhibit autophagy by modulating a PTEN/Akt signaling pathway (12). miR-221 targeting PTEN/Akt is also reported in several cancer cells during cancer development (33-35). Our results implicated a potential role of TP53INP1 on the effect of miR-221 on autophagy of CRC.

In conclusion, our findings explore a new role of miR-221 in autophagy regulation of cancer. The tumor promotion function of miR-221 in CRC could be the result of autophagy inhibition. TP53INP1 is a new target of miR-221 in CRC cells. Although further experiments should be performed, our data suggested that the effect of miR-221 on autophagy might be mediated by TP53INP1.

\section{Acknowledgements}

This study was supported by the Science and Technology Planning Project of Guangdong Province (grant no. 2013B021800070), the Training Plan for Outstanding Young Teachers in Higher Education Institutions of Guangdong Province (grant no. YQ201403/YQ2014086), the Hunan Provincial Natural Science Foundation of China (grant no. 2015jj6066), and the Project of Hunan Provincial Department of Health (grant no. B2012-095).

\section{References}

1. Favoriti P, Carbone G, Greco M, Pirozzi F, Pirozzi RE and Corcione F: Worldwide burden of colorectal cancer: A review. Updates Surg 68: 7-11, 2016.

2. Jemal A, Bray F, Center MM, Ferlay J, Ward E and Forman D: Global cancer statistics. CA Cancer J Clin 61: 69-90, 2011.

3. Ferrari P, Jenab M, Norat T, Moskal A, Slimani N, Olsen A, Tjønneland A, Overvad K, Jensen MK, Boutron-Ruault MC, et al: Lifetime and baseline alcohol intake and risk of colon and rectal cancers in the European prospective investigation into cancer and nutrition (EPIC). Int J Cancer 121: 2065-2072, 2007.
4. Burada F, Nicoli ER, Ciurea ME, Uscatu DC, Ioana M and Gheonea DI: Autophagy in colorectal cancer: An important switch from physiology to pathology. World J Gastrointest Oncol 7: 271-284, 2015.

5. Liu B, Wen X and Cheng Y: Survival or death: Disequilibrating the oncogenic and tumor suppressive autophagy in cancer. Cell Death Dis 4: e892, 2013.

6. Zhai H, Fesler A and Ju J: MicroRNA: A third dimension in autophagy. Cell Cycle 12: 246-250, 2013.

7. An Y, Zhang Z, Shang Y, Jiang X, Dong J, Yu P, Nie Y and Zhao Q: miR-23b-3p regulates the chemoresistance of gastric cancer cells by targeting ATG12 and HMGB2. Cell Death Dis 6: e1766, 2015.

8. Jin F, Wang Y, Li M, Zhu Y, Liang H, Wang C, Wang F, Zhang CY, Zen K and Li L: MiR-26 enhances chemosensitivity and promotes apoptosis of hepatocellular carcinoma cells through inhibiting autophagy. Cell Death Dis 8: e2540, 2017.

9. Nyhan MJ, O'Donovan TR, Boersma AW, Wiemer EA and McKenna SL: MiR-193b promotes autophagy and non-apoptotic cell death in oesophageal cancer cells. BMC Cancer 16: 101, 2016.

10. Su M, Wang J, Wang C, Wang X, Dong W, Qiu W, Wang Y, Zhao X, Zou Y, Song L, et al: MicroRNA-221 inhibits autophagy and promotes heart failure by modulating the p27/CDK2/mTOR axis. Cell Death Differ 22: 986-999, 2015.

11. Chen Q, Zhou Y, Richards AM and Wang P: Up-regulation of miRNA-221 inhibits hypoxia/reoxygenation-induced autophagy through the DDIT4/mTORC1 and Tp53inp1/p62 pathways. Biochem Biophys Res Commun 474: 168-174, 2016.

12. Li L, Wang Z, Hu X, Wan T, Wu H, Jiang W and Hu R: Human aortic smooth muscle cell-derived exosomal miR-221/222 inhibits autophagy via a PTEN/Akt signaling pathway in human umbilical vein endothelial cells. Biochem Biophys Res Commun 479: 343-350, 2016.

13. Pradhan AK, Talukdar S, Bhoopathi P, Shen XN, Emdad L, Das SK, Sarkar D and Fisher PB: mda-7/IL-24 mediates cancer cell-specific death via regulation of miR-221 and the Beclin-1 Axis. Cancer Res 77: 949-959, 2017.

14. Chen WX, Hu Q, Qiu MT, Zhong SL, Xu JJ, Tang JH and Zhao JH: miR-221/222: Promising biomarkers for breast cancer. Tumour Biol 34: 1361-1370, 2013.

15. Sancho A, Duran J, García-España A, Mauvezin C, Alemu EA, Lamark T, Macias MJ, DeSalle R, Royo M, Sala D, et al: DOR/Tp53inp2 and Tp53inp1 constitute a metazoan gene family encoding dual regulators of autophagy and transcription. PLoS One 7: e34034, 2012.

16. Tomczak K, Czerwińska P and Wiznerowicz M: The cancer genome atlas (TCGA): An immeasurable source of knowledge. Contemp Oncol (Pozn) 19: A68-A77, 2015.

17. Tanida I, Minematsu-Ikeguchi N, Ueno T and Kominami E: Lysosomal turnover, but not a cellular level, of endogenous LC3 is a marker for autophagy. Autophagy 1: 84-91, 2005.

18. Lewis BP, Burge CB and Bartel DP: Conserved seed pairing, often flanked by adenosines, indicates that thousands of human genes are microRNA targets. Cell 120: 15-20, 2005.

19. Enright AJ, John B, Gaul U, Tuschl T, Sander C and Marks DS: MicroRNA targets in Drosophila. Genome Biol 5: R1, 2003.

20. Loher P and Rigoutsos I: Interactive exploration of RNA22 microRNA target predictions. Bioinformatics 28: 3322-3323, 2012.

21. Garofalo M, Quintavalle C, Romano G, Croce CM and Condorelli G: miR221/222 in cancer: Their role in tumor progression and response to therapy. Curr Mol Med 12: 27-33, 2012.

22. Volinia S, Calin GA, Liu CG, Ambs S, Cimmino A, Petrocca F, Visone R, Iorio M, Roldo C, Ferracin M, et al: A microRNA expression signature of human solid tumors defines cancer gene targets. Proc Natl Acad Sci USA 103: 2257-2261, 2006.

23. Garofalo M, Quintavalle C, Di Leva G, Zanca C, Romano G, Taccioli C, Liu CG, Croce CM and Condorelli G: MicroRNA signatures of TRAIL resistance in human non-small cell lung cancer. Oncogene 27: 3845-3855, 2008.

24. Gottardo F, Liu CG, Ferracin M, Calin GA, Fassan M, Bassi P, Sevignani C, Byrne D, Negrini M, Pagano F, et al: Micro-RNA profiling in kidney and bladder cancers. Urol Oncol 25: 387-392, 2007.

25. Pu XX, Huang GL, Guo HQ, Guo CC, Li H, Ye S, Ling S, Jiang L, Tian Y and Lin TY: Circulating miR-221 directly amplified from plasma is a potential diagnostic and prognostic marker of colorectal cancer and is correlated with p53 expression. J Gastroenterol Hepatol 25: 1674-1680, 2010. 
26. Gugnoni M, Sancisi V, Manzotti G, Gandolfi G and Ciarrocchi A: Autophagy and epithelial-mesenchymal transition: An intricate interplay in cancer. Cell Death Dis 7: e2520, 2016.

27. Mathew R, Karantza-Wadsworth V and White E: Role of autophagy in cancer. Nat Rev Cancer 7: 961-967, 2007.

28. Kroemer G, Mariño G and Levine B: Autophagy and the integrated stress response. Mol Cell 40: 280-293, 2010.

29. Seillier M, Peuget S, Gayet O, Gauthier C, N'Guessan P Monte M, Carrier A, Iovanna JL and Dusetti NJ: TP53INP1, a tumor suppressor, interacts with LC3 and ATG8-family proteins through the LC3-interacting region (LIR) and promotes autophagy-dependent cell death. Cell Death Differ 19: 1525-1535, 2012.

30. Wang W, Liu J and Wu Q: MiR-205 suppresses autophagy and enhances radiosensitivity of prostate cancer cells by targeting TP53INP1. Eur Rev Med Pharmacol Sci 20: 92-100A, 2016.

31. Xu CG, Yang MF, Fan JX and Wang W: MiR-30a and miR-205 are downregulated in hypoxia and modulate radiosensitivity of prostate cancer cells by inhibiting autophagy via TP53INP1. Eur Rev Med Pharmacol Sci 20: 1501-1508, 2016.
32. Kim J, Jiang J, Badawi M and Schmittgen TD: miR-221 regulates CD44 in hepatocellular carcinoma through the PI3K-AKT-mTOR pathway. Biochem Biophys Res Commun 487: 709-715, 2017.

33. Li B, Lu Y, Wang H, Han X, Mao J, Li J, Yu L, Wang B, Fan S, Yu X and Song B: miR-221/222 enhance the tumorigenicity of human breast cancer stem cells via modulation of PTEN/Akt pathway. Biomed Pharmacother 79: 93-101, 2016.

34. Li J, Yao L, Li G, Ma D, Sun C, Gao S, Zhang P and Gao F: miR-22 promotes epithelial-mesenchymal transition through targeting PTEN and forms a positive feedback loop with $\beta$-catenin/c-Jun signaling pathway in extra-hepatic cholangiocarcinoma. PLoS One 10: e0141168, 2015.

35. Yang W, Yang Y, Xia L, Yang Y, Wang F, Song M, Chen X, Liu J, Song Y, Zhao Y and Yang C: MiR-221 promotes capan-2 pancreatic ductal adenocarcinoma cells proliferation by targeting PTEN-Akt. Cell Physiol Biochem 38: 2366-2374, 2016. 